\title{
Assessment of Liver Transplant Grafts on Gadoxetic Acid Enhanced-MRI in Pediatric Patients
}

\author{
Gungoren FZ ${ }^{1,2}$, Hodge J C ${ }^{1}$, Hayat $\mathrm{A}^{1,3}$, Bastati $\mathrm{N}^{1}$, \\ Gehan $\mathbf{M}^{4}$ and Ba-Ssalamah $\mathbf{A}^{1 *}$ \\ ${ }^{1}$ Department of Biomedical Imaging and Image-guided \\ Therapy, Medical University Vienna, Austria \\ ${ }^{2}$ Department of Radiology, Istanbul Medipol University, \\ Turkey \\ ${ }^{3}$ Department of Radiology, IBN Sina Hospital, Kuwait \\ ${ }^{4}$ Department of Pediatrics and Adolescent Medicine, \\ Medical University Vienna, Austria \\ *Correspondling author: Ba-Ssalamah A, Department \\ of Biomedical Imaging and Image-guided Therapy, \\ Medical University Vienna, Währinger Guertel 18-20A- \\ 1090, Vienna, Austria
}

Received: March 28, 2017; Accepted: May 15, 2017; Published: May 25, 2017

\begin{abstract}
A wide spectrum of neonatal and pediatric liver diseases can be successfully treated by liver Transplantation (LT). During the last sixty years, refined diagnostic and surgical techniques, improvements in perioperative care, and the advent of better immunosuppressive medications have established LT as a viable treatment option for acute and chronic liver failure in children. As clinical and laboratory findings of LT complications are often nonspecific, imaging tests play a major role in both diagnosis and guidance of therapeutic interventions. In this article, we wanted to emphasize the potential role of gadoxetic acid-enhanced $M R I$ in the evaluation of liver transplant grafts in the pediatric population.We included seven pediatric patients who underwent gadotexic acid-enhanced MRI with different operative procedures.
\end{abstract}

Our series suggests potentially added value of gadoxetic acid for the evaluation of morphologic LT complications, i.e., vascular, biliary, or anastomotic obstruction, as well as parenchymal disorders, including global or segmental (for example, auxiliary LT) hepatobiliary dysfunction.

Gadoxetic acid-enhanced MRI showed its ability to depict morphological or anatomical postoperative complications, including vascular occlusion or biliary strictures. Contrary to the conventional imaging modalities or nuclear medicine exams, gadoxetic acid-enhanced MRI can also provide us simultaneously with global and segmental functional information, with high spatial resolution and no radiation exposure.

In this article, we show our imaging experience using gadoxetic acidenhanced MRI in the evaluation of LT in children, and its superiority to US, $\mathrm{CT}$, and conventional MRI, by providing anatomic and functional data about the graft.

Keywords: MRI; Contrast material; Liver; Pediatric

\section{Case Presentation}

Liver Transplantation (LT) is the only curative treatment of acute or chronic liver failure in pediatric patients [1]. However, contrary to adults, pediatric LT may be performed using a variety of different techniques, according to the indication, graft availability and donorrecipient size match [2]. In kids, a subtotal or total liver graft may be transplanted from a living or deceased donor, respectively. Furthermore, the graft can either replace or be added to the native liver. In the latter case, the so-called auxiliary LT supports the child until his native liver recovers from acute failure [3]. To interpret post-operative images, the radiologist must know the type of LT procedure done, as the complications and prognosis vary with the type of surgery. Although US, and less often angiography and ERCP, have been used to study morphologic complications of the graft post-operatively, until recently, there has been no non-invasive, operator-independent imaging modality to do this [4]. CT remains less than ideal, in the pediatric population, due to its ionizing radiation. Conventional MRI, unlike gadoxetic acid enhanced-MRI, gives only morphologic information, i.e. pertaining to vascular and biliary anastomoses [5]. Using gadoxetic acid enhanced-MRI, however, we are able to obtain information regarding liver function, too. Furthermore, using this specific Contrast Media (CM), we can detect global and segmental parenchymal dysfunction due its unique pharmacokinetic properties [6]. This CM is used routinely in adults and off-label in children, for the work-up of focal liver lesions, diffuse liver diseases and hepatobiliary disorders, including the evaluation of LT grafts [7]. Through the following case series, we illustrate the use of gadoxetic acid enhanced-MRI in a variety of post-op LT patients with a broad spectrum of complications demonstrating its morphologic and functional properties.

\section{Case 1}

A 14-year-old boy with known Ulcerative Colitis (UC), Primary Sclerosing Cholangitis (PSC) and Autoimmune Hepatitis (AIH) was brought to the ED with abdominal pain. Laboratory tests demonstrated severe elevation of cholestatic liver function parameters. MRCP performed preoperatively showed a "string of pearls", i.e. irregularity of the bile duct caliber, very characteristic of PSC (Figure 1a). Gadoxetic acid enhanced-MRI showed diminished uptake and absent excretion of $\mathrm{CM}$ in the liver on HBP images consistent with end-stage chronic liver disease (Figure 1b,c). Soon thereafter, he received a whole liver graft, i.e. orthotopic LT. Fivemonths post-op MRI showed good uptake and timely excretion of 


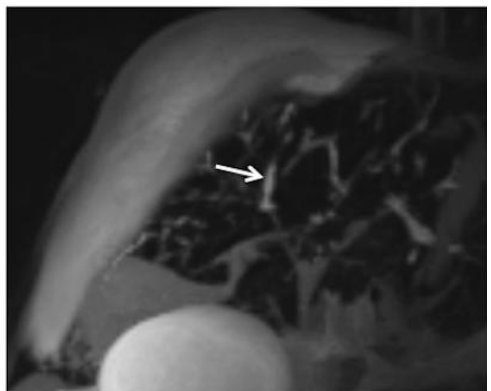

Figure 1a: MRCP performed preoperatively showing 'string of pearls' sign with irregular intrahepatic bile duct caliber characteristic of advanced PSC (arrow).

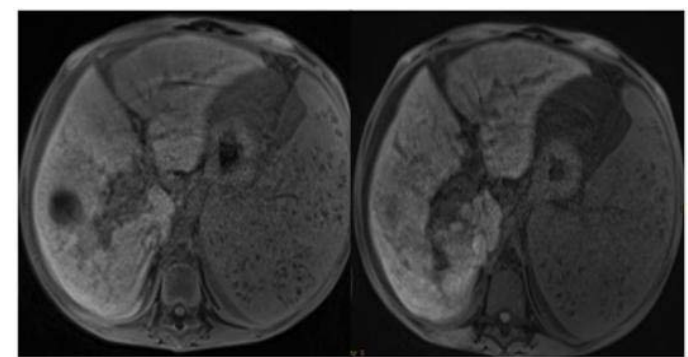

Figure 1b,c: Preoperative gadoxetic acid enhanced-MRI scan shows almost no uptake and no excretion of $\mathrm{CM}$ on the HBP image (1c) in comparison with the non contrast image (1b) reflecting severe hepatic dysfunction.

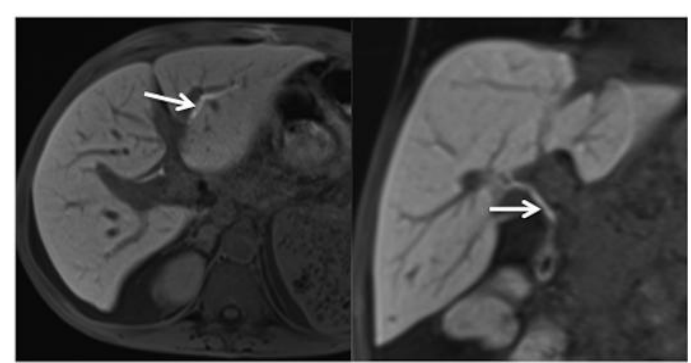

Figure 1d,e: Axial and coronal gadoxetic-acid enhanced-MR scan fivemonths post-transplant showing normal uniform CM uptake and timely excretion (arrows) of the graft consistent with normal function.

CM in the HBP (Figure 1d,e). Laboratory tests at both time points also confirmed normal liver function.

\section{Case 2}

A 7-month-old infant was brought to the Emergency Department (ED) with confusion. Physical examination was unremarkable except for signs of encephalopathy. Liver enzymes, including ammonia level, were elevated indicating acute liver failure. A living-related donor LT, for acute hepatic necrosis, was performed immediately, using the mother's left lobe as the graft. Postoperatively, the patient was placed on ursodeoxycholic acid, tacrolimus and cortisone. The patient was fine until 10 years later, when cortisone therapy was discontinuedto reduce its long-term side effects. US and subsequent abdominal CT were performed for mild cholestasis. Although the intrahepatic bile ducts were not significantly dilated on CT scan, LFTs were elevated arousing suspicion of biliary anastomotic stenosis proximal to the Common Bile Duct (CBD). Gadoxetic acid-enhanced MRI was

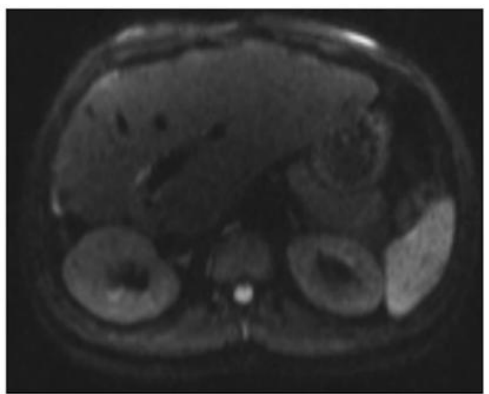

Figure 2a: DWI image 10 years after liver transplantation. The graft is unremarkable.

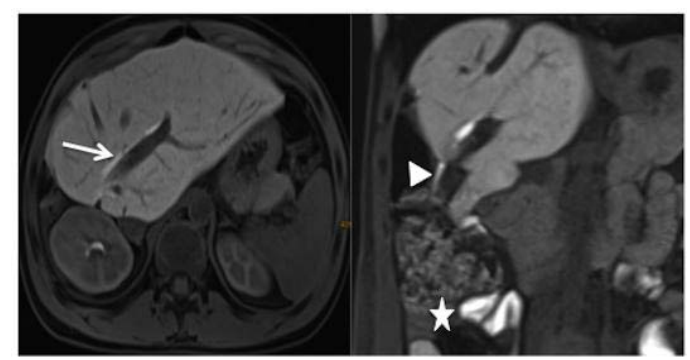

Figure 2b,c: Ten years post-transplantation gadoxetic acid enhanced-MR with normal graft function. HBP axial and coronal images demonstrate normal uptake (2b) and timely excretion of this hepatospecific CM into the bile duct (arrow) and ultimately the hepatojejunostomy anastomosis (arrowhead) excluding mechanical obstruction (2c). Note: CM accumulation in the small bowel (asterisk).

performed to rule out mechanical obstruction. MRCP and DWI showed no bile duct dilatation (Figure 2a). The Hepatobiliary Phase (HBP) images, after 20 minutes, showed normal CM uptake and excretion in the graft. No narrowing was identified within the biliary tree (Figure $2 \mathrm{~b}, \mathrm{c}$ ). The patient is still alive 12 years after the operation without any complaints.

\section{Case 3}

An 11-year-old boy was brought to the ED with low-grade fever and abdominal pain. His medical history was remarkable for a renal and whole LT a year ago for Autosomal Dominant Polycystic Kidney Disease (ADPKD). A few months post-op, he returned with fever and septicemia. An infected bileleak was diagnosed and drained at CT. Eight months after LT, a $2 \mathrm{~cm}$ biloma, as well as moderate cholangiectasis were identified on MRCP (Figure 3a). DWI images showed segmental

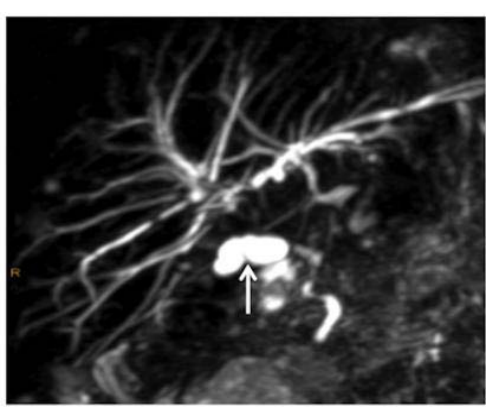

Figure 3a: Eight months postoperatively coronal MRCP image shows a $2 \mathrm{~cm}$ fluid collection (arrow) near the anastomosis, consistent with a biloma. 


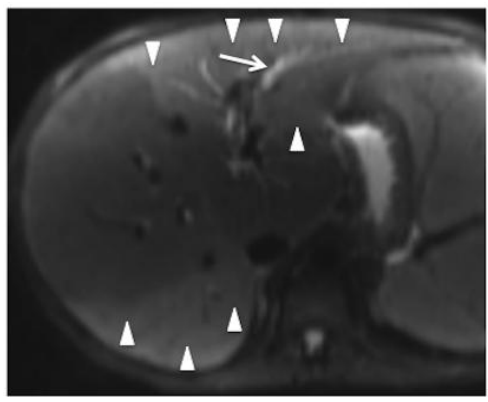

Figure 3b: DWI images 8 months postoperatively showing moderate cholangiectasis (arrow) and edema within the left and right (segment VII) liver lobes (arrowheads). This ascending cholangitis occurs via hepatojejunostomy.

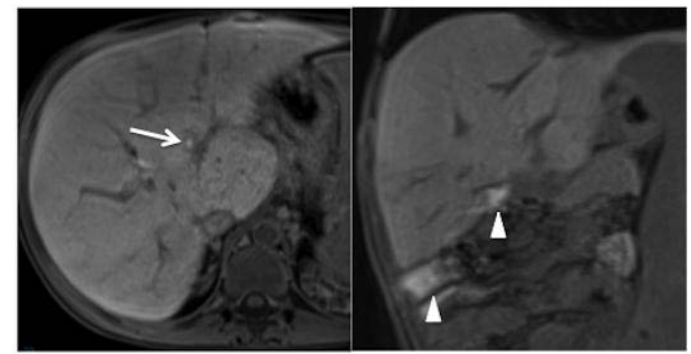

Figure 3c,d: Gadoxetic acid enhanced-MRI images after revision. HBP axial (3c) and coronal (3d) images illustrate heterogeneous uptake but timely excretion of $\mathrm{CM}$ into the bile ducts (arrow). CM enters the bowel via the hepatojejunostomy (arrowheads)

liver edema indicating ascending cholangitis (Figure 3b). Moreover, the HBP images showed poor uptake and no excretion of CM even after 2 hours post-injection. A revision of the biliary anastomosis was performed. Intra-operative liver biopsy confirmed the diagnosis of cholangitis and progressive cirrhosis. Gadoxetic acid enhancedMRI performed after revision showed heterogeneous CM uptake but timely excretion via the hepatojejunostomy (Figure $3 \mathrm{c}, \mathrm{d}$ ). The patient has been fine on tacrolismus since the revision, with normal LFTs.

\section{Case 4}

A 6-year-old boy with non metastatichepatoblastoma underwent a living-related LT, segments II-IV from his father's liver serving as the graft. He was placed on Cyclosporineimmunosuppression. At post-op week 5, noncholestatic LFTs were mildly elevated.

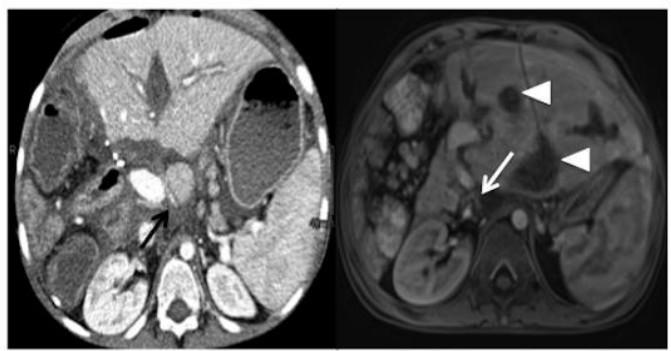

Figure 4a,b: CT scan perfomed 5 weeks after transplantation shows pre stenotic dilation of the hepatic artery proximal to the stenosis (black arrow) near the surgical clips (4a). Two months post-transplantation there is nonvisualization of the hepatic artery at the celiac trunk on contrast-enhanced MRI, portal venous phase, arousing suspicion of hepatic artery stenosis ( arrow in $4 b$ ). Multiple bilomas are depicted (arrowheads in 4b).

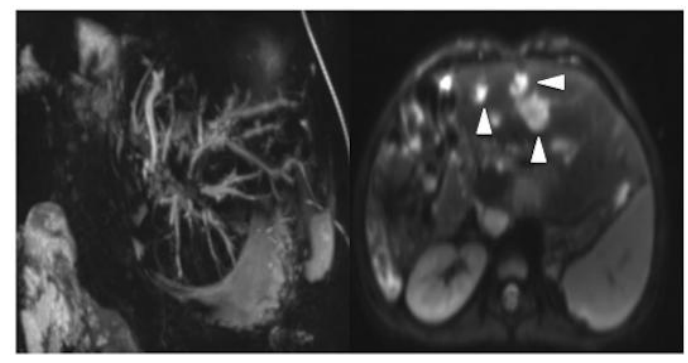

Figure 4c,d: Two months post-operative coronal T2 MRCP MIP image (4c) shows moderately dilated intrahepatic bile ducts in both liver lobes. The diffusion-weighted image (4d) demonstrates multiple scattered high signal intensity fluid collections which arise from the biliary tree, characteristic of ischemic-related cholangitis (arrowheads in 4d).

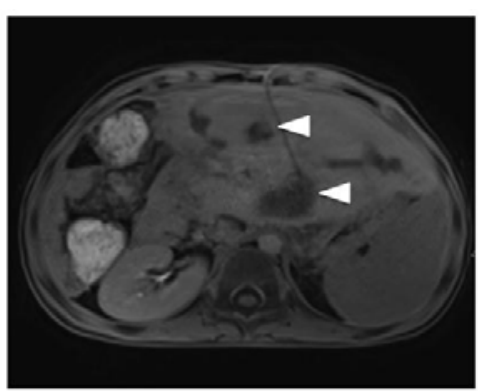

Figure 4e: An axial HBP image, 2 months postop shows reduced uptake of $\mathrm{CM}$ in the graft, compatible with graft dysfunction. CM was seen in the graft on delayed images (not shown). Multiple bilomas are to be seen (arrowheads).

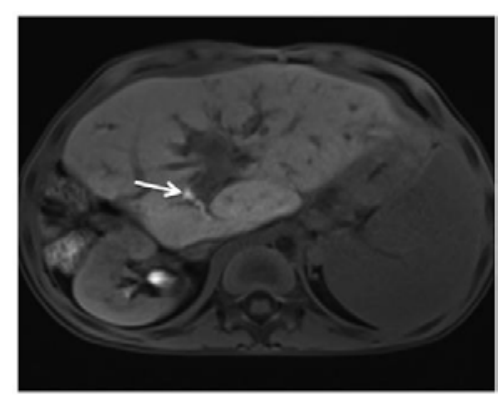

Figure 4f: Twenty-one months status post reduced-size cadaveric live transplantation. Gadoxetic acid enhanced-MRI, axial HBP image, shows good uptake and timely excretion (arrow) of CM in the graft representing good graft function.

Subsequent color Doppler showed no flow in the Hepatic Artery (HA). Emergency CT angiography confirmed the HA occlusion (Figure 4a) and also showed multiple hypodense lesions in the liver compatible with ischemic necrosis/biloma. The HA could only be partially recanalized during angiography. Gadoxetic acid enhancedMRI, 2 months later, showed partial HA stenosis (Figure 4b). MRCP and DWI, during the same imaging session, showed moderately dilated intrahepatic bile ducts and scattered bilomas in the liver graft, which appeared to be constant (Figure 4c,d). Additionally, the HBP images showed reduced uptake and delayed excretion of CM in the graft, compatible with graft dysfunction (Figure 4e). The child was waitlisted for a second LT. Nine months later, he received a reducedsize cadaveric LT of segments I-IV. The most recent MRI, 21 months 


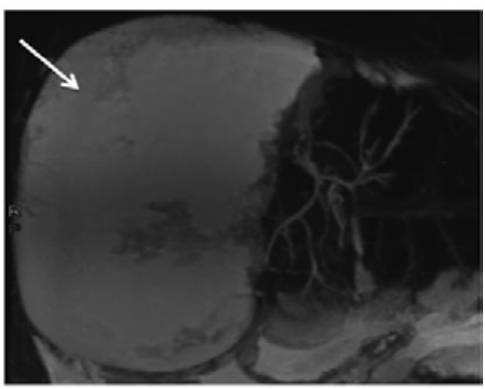

Figure 5a: MRCP 6 weeks after liver transplantation shows a huge extrahepatic fluid collection which was proven to be a biloma (arrow).

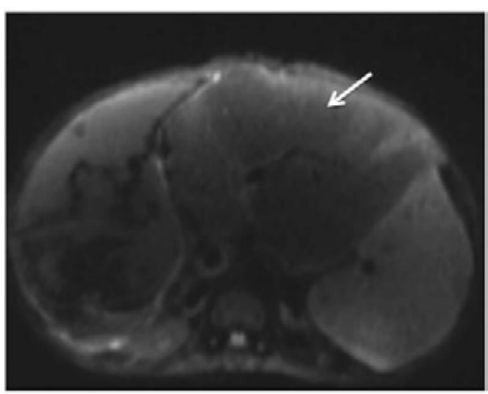

Figure 5b: DWI sequence, 6 weeks post-op, shows a sharply defined area of altered signal intensity in segments II and III compatible with segmental cholangitis (arrow)

after cadaveric LT, showed normal uptake and excretion on the HBP (Figure 4f).

\section{Case 5}

A 9-year-old boy was brought to the ED with shortness of breath. As an infant he had undergone a Kasai operation for biliary atresia. Admission physical exam was unremarkable except for dyspnea. He was diagnosed with hepatopulmonary syndrome after his blood work showed mildly elevated liver enzymes (noncholestatic and cholestatic). Soon after, he underwent a living donor LT, receiving his father's segments II, III, and IV as a graft. Ten days post-op, he developed thrombocytopenia, and suffered a seizure. A massive intracerebral hematoma, confirmed on CT, was evacuated. During post-op week 2, US done for peritoneal signs, demonstrated portal vein

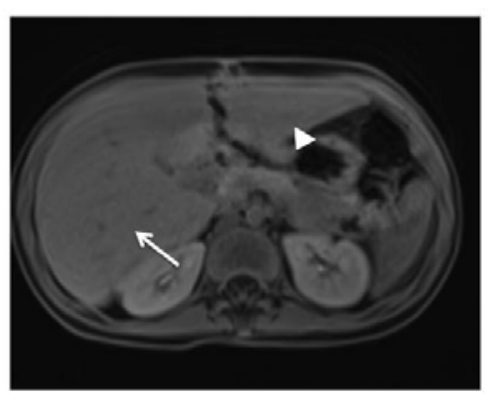

Figure 5c: HBP images, 6 weeks after transplantation. There is a large low signal intensity fluid collection (arrow) consistent with a biloma. The 20 minute image shows diminished $\mathrm{CM}$ uptake and absent excretion in the liver graft consistent with severe graft dysfunction. Delayed images were not obtained as the patient was anesthetized for the exam. Therefore, the excretion of $\mathrm{CM}$ into the biloma is not depicted.

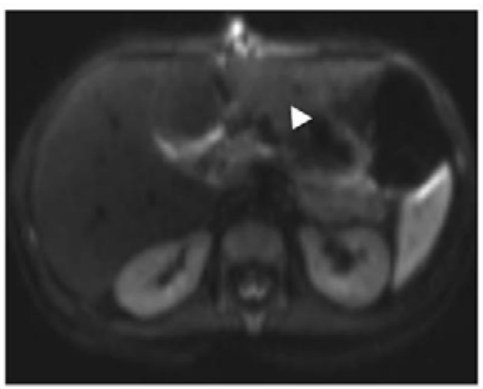

Figure 6a: One month status post auxiliary liver transplantation. MRI axial HBP, both graft (arrowhead) and native liver (arrow) segments show diminished gadoxetic acid uptake and reduced excretion. Again, delayed images, i.e. beyond 20 minutes, were not obtained to avoid prolonging anesthetization of the child.

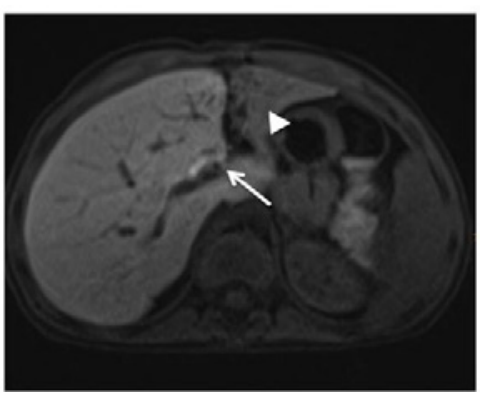

Figure 6b: Axial DWI shows diffuse hyper intense signal in the left lobe graft compatible with edema due to rejection (arrowhead). The native right liver is unremarkable.

thrombosis and free intraperitoneal fluid. Gadoxetic acid enhancedMRI, during post-op week 6, showed segmental cholangitis and a giant extra-hepatic fluid collection (Figure 5a,b). HBP images showed diminished CM uptake and no excretion of the liver graft consistent with severe graft dysfunction (Figure 5c). A bile leak was confirmed upon puncturing the collection. The patient underwent revision of the biliary anastomosis and correction of the choledochal fistula. Two months post-LT, the patient passed away due to multiple organ failure and massive cerebral hemorrhage caused by hypersplenisminduced thrombocytopenia.

\section{Case 6}

A two-year old patient was brought to our ED with confusion. His medical history was remarkable for a diagnosis of citrullinemia type I for which he had undergone liver cell transplantation as a bridge to LT at 9 months old. He underwent a reduced-size LT for hyperammonemia some days later. An auxiliary left lobe was placed beside his preserved right liver lobe. On initial MRI scan, at post-op month 1, both graft and native liver segments showed diminished gadoxetic acid uptake and no excretion on HBP images (Figure 6a). Moreover mild edema of the auxiliaryliver was noted (Figure $6 \mathrm{~b}$ ). MRI was again done during post-op month 6 for repeat hyperammonemia. DWI showed edema and cholangiectasis in the left liver graft due to segmental cholangitis. HBP images showed normal CM uptake with delayed excretion in the auxiliary graft but good CM uptake and excretion in the native liver (Figure 6c). Unfortunately the patient expired from a cardiac arrest. The autopsy report ruled out rejection. 


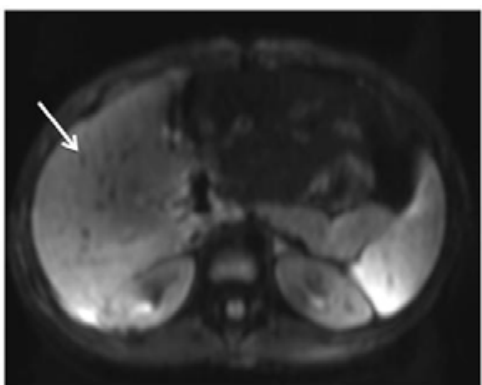

Figure 6c: Post-op 6 months- An axial MRI, HBP, shows regular uptake and timely excretion of the native right liver lobe (arrow) but diminished uptake and no excretion from the auxiliary left lobe due to rejection (arrowhead). This example demonstrates the value of gadoxetic acid enhanced-MRI to evaluate global and segmental function of the liver.

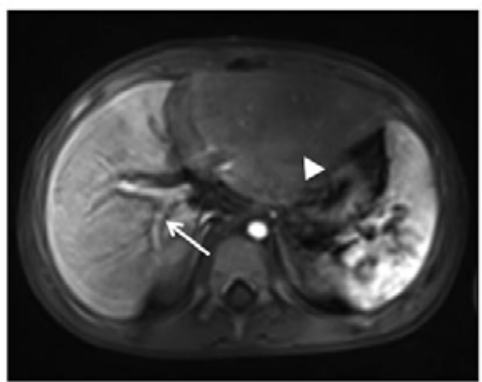

Figure 7a: A week after auxiliary liver transplantation. DWI shows diffuse high signal intensity in the native right lobe consistent with acute hepatitis (arrow). The signal in the left lobe graft is suggestive of periportal tracking.

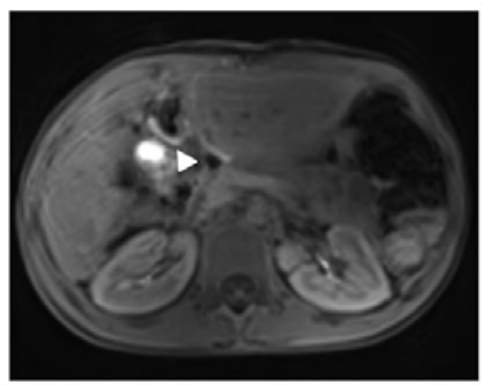

Figure 7b: Gadoxetic acid enhanced-MRI, arterial phase, a week after surgery shows above-normal CM uptake in the native right liver lobe (arrow) whereas the left lobe graft appears hypo intense due to severe dysfunction (arrow head).

\section{Case 7}

A 6-year-old boy was brought to the ED with drowsiness. Labs showed markedly elevated LFTs, as well as a mild coagulopathy and hyperammonemia accounting for his hepatic encephalopathy. A tru-cut liver biopsy was remarkable for acute liver failure caused by autoimmune hepatitis. The patient immediately underwent a livingrelated auxiliary LT, receiving his father's left lobe where the native right lobe of the patient was preserved. A week post-LT, his ammonia and GGT levels were again elevated. MRI with DWI showed edema in his native right liver lobe (Figure 7a). Furthermore, dynamic gadoxetic acid imaging was remarkable for hyperemia in the native right liver lobe. Vascular complications were excluded on review of

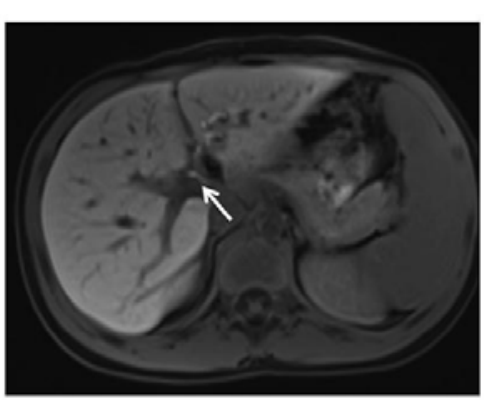

Figure 7c: A week after transplantation an axial MRI, HBP, shows heterogeneous $\mathrm{CM}$ uptake in both lobes. There is excretion into the hepatojejunostomy from the left liver graft after 180 minutes (arrowhead), but no excretion from the native right liver

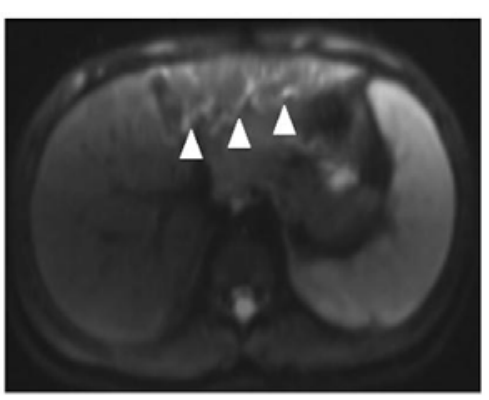

Figure 7d: Axial MRI, HBP, done 16 months postoperatively. Both right lobe native and left lobe graft show normal CM uptake and timely excretion (arrow) indicating normal liver function.

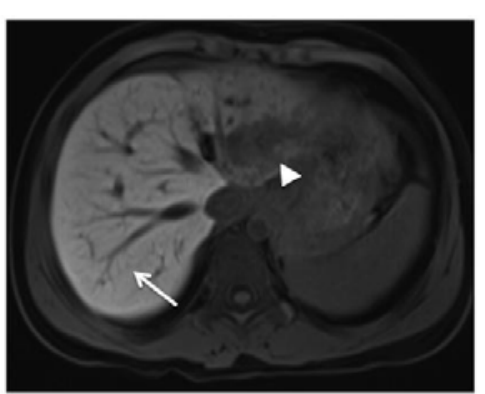

Figure 7e: Axial MRI at post-op year two, after further immunosuppressant reduction. The DWI shows cholangiectasis and diffuse edema in the anterior part of the left liver graft (arrow heads) due to rejection.

arterial and portal venous phase images (Figure $7 \mathrm{~b}$ ). Furthermore, CM uptake and excretion on HBP images were absent consistent with severe dysfunction of the native liver. The auxiliary left lobe showed diminished CM uptake and delayed excretion in the HBP suggestive of post-operative delayed function (Figure 7c). The child was placed on tacrolismus. His course improved dramatically at post-LT month 16, at which time gadoxetic acid enhanced-MRI depicted regular function of both auxiliary and native liver (Figure 7d). The clinician decided to reduce immunosuppression slowly since the native liver was working again. A follow-up MR at post-op year two, after further immunosuppressant reduction, showed cholangiectasis and diminished function in the auxiliary left lobe (Figure 7e), likely due to chronic rejection. This rejection was of no clinical concern since the native host liver had good function. In the third post-operative year, 


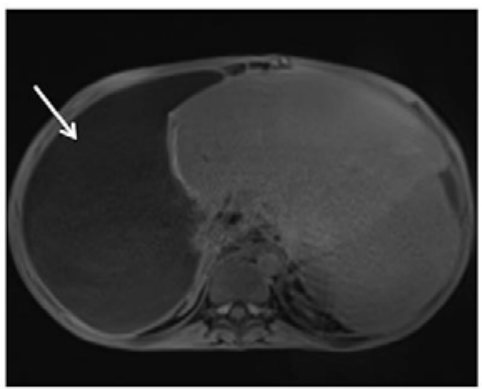

Figure 7f: Thirty months after transplantation, the axial HBP shows decreased $\mathrm{CM}$ uptake and shrinkage of left liver lobe (arrow head) due to chronic rejection. A 90 minute image, not shown, illustrated delayed excretion. Note that the native right lobe shows normal uptake (arrow) on this 20 minute HBP image.

immunosupressants were discontinued. Gadoxetic acid enhancedMRI, at post-op 30 month showed normal function of his native lobe and continued worsening function of the auxiliary lobe (Figure 7f). Today the patient is alive and well, his LFTs within normal limits.

\section{Discussion/Conclusion}

Pediatric LT is the treatment of choice for acute liver failure and end-stage chronic liver disease.Because liver grafts are scarce and LT is exceedingly expensive, close patient follow-up is imperative to give the graft the best chance of survival [8,9]. Early diagnosis of graft dysfunction, whether acute or chronic, should be made as soon as possible to allow early intervention [10,11]. Imaging usually begins with Ultrasonography (US) and Doppler, as they are most practical, done at the patient's bedside, in the early post-op period $[4,12,13]$. Suspected vascular complicationsare usually confirmed by CT and/or CT angiography $[5,14,15]$. Biliary complications may be confirmed by $\mathrm{CT}$ as well as contrast-enhanced Magnetic Resonance Imaging (MRI) and MRCP [15]. In the pediatric population, conventional MRI would be preferable to CT to avoid ionizing radiation [16]. However, if parenchymal graft dysfunction, i.e. rejection, recurrent disease, is the clinical question, then gadoxetic acid enhanced-MRI would be the exam of choice as it can exclude morphologic complications, such as vascular and biliary disorders, and, detect parenchymal dysfunction simultaneously [17]. In our case series we demonstrated gadoxetic acid's role in evaluation of pre- and post- transplant diagnostic workup. In the first patient, gadoxetic acid-enhanced MRI showed neither uptake nor excretion due to end-stage cholestasis of PSC, but good function after LT, similar to patient 2. Furthermore, gadoxetic acid-enhanced MRI simultaneously depicted both the vascular and biliary structures [cases 3,4], as well as graft dysfunction - both global [case 5] and segmental, i.e. auxiliary LT [cases 6,7]. A variety of different methods for quantitative assessment of global liver function are available, including clearance-retention tests and nuclear medicine methods [18]. Whereas the former serologic tests can not provide data about regional function, the latter technique exposes the patient to radiation and has low spatial resolution.

Gadoxetic acid enhanced-MRI can also help predict liver graft survival, as shown by Wibmer et al. [17] and more recently by Bastati et al [19]. They demonstrated that patients with normal gadoxetic acid uptake and excretion had a $100 \%$ probability of one-to three-year re transplantation-free survival, whereas more than half of the patients (in the absence of mechanical bile duct obstruction) with impaired uptake and/or excretion died or had to undergo re-transplantation within one year [17]. These facts show the impact of this noninvasive imaging tool to evaluate structural and/or parenchymal LTassociated complications. These results can be confirmed in our case series. Patients 1 and 2, despite vascular and biliary complications, had good uptake and punctual excretion and thus survived. However patient 4 , with poor uptake and excretion had to be re-transplanted due to graft failure.

To understand why gadoxetic acid enhanced-MRI gives us functional information, we consider its pharmacokinetics and how it differs from conventional gadolinium CM. Gadoxetic acidhas extracellular (nonspecific) and intracellular (hepatocyte-specific) features [20], with both renal (50\%) and biliary (50\%) excretion pathways. The uptake and biliary excretion pathway are mediated through ATP-dependent glutathione-S-transferases, OATP andMRP2/3, respectively [21]. Hepatic uptake and excretion with gadoxetic acid peaks 20 minutes after administration, although it remains within the hepatobiliary system for more than 120 minutes [22]. The vast majority of clinical experience with gadoxetic acid is within the adult population $[23,24]$. As the pediatric population has distinct pathophysiological processes, all studies- including our recent report- describe the off-label use of gadoxeticacid in those less than 18 years of age. Although, to date, the FDA has not approved its use in children, the large body of literature citing its use in this group suggests that it is safe in children, too [25-29]. Experience has shown that gadoxetic acid's safety profile is similar to that of other gadolinium-based CM. Furthermore, they have found better diagnostic performanceof gadoxetic acid without a higher incidence of adverse reactions $[7,30,31]$. We can confirm these results in this small cohort and a larger adult cohort of OLT and non-transplant patients examined at our center.

We report our initial experience with gadoxetic acid in pediatric $\mathrm{LT}$ recipients. This is the first case series with this hepatocyte-specific MR agent in the pediatric population. Our series suggests potentially added value of gadoxetic acidfor evaluation of morphologic LT complications, i.e. vascular, biliary or anastomotic obstruction, as well as parenchymal disorders, including global or segmental (for e.g. auxiliary LT) hepatobiliary dysfunction.

\section{References}

1. Abbasoglu O. Liver transplantation: yesterday, today and tomorrow. World J Gastroenterol. 2008; 14: 3117-3122.

2. Hackl C, Schlitt HJ, Melter M, Knoppke B, Loss M. Current developments in pediatric liver transplantation. World J Hepatol. 2015; 7: 1509-1520.

3. Azouz SM, Diamond IR, Fecteau A. Graft type in pediatric liver transplantation. Curr Opin Organ Transplant. 2011; 16: 494-498.

4. Friedewald SM, Molmenti EP, DeJong MR, Hamper UM. Vascular and nonvascular complications of liver transplants: sonographic evaluation and correlation with other imaging modalities and findings at surgery and pathology. Ultrasound Q. 2003; 19: 71-85.

5. Ito K, Siegelman ES, Stolpen AH, Mitchell DG. MR imaging of complications after liver transplantation. AJR Am J Roentgenol. 2000; 175: 1145-1149.

6. Ba-Ssalamah A, Qayyum A, Bastati N, Fakhrai N, Herold CJ, Caseiro Alves F. P4 radiology of hepatobiliary diseases with gadoxetic acid-enhanced MRI as a biomarker. Expert Rev Gastroenterol Hepatol. 2014; 8: 147-160.

7. Tamrazi A, Vasanawala SS. Functional hepatobiliary MR imaging in children 
Pediatr Radiol. 2011; 41: 1250-1258.

8. van der Hilst CS, ljtsma AJ, Slooff MJ, Tenvergert EM. Cost of liver transplantation: a systematic review and meta-analysis comparing the United States with other OECD countries. Med Care Res Rev. 2009; 66: 3-22.

9. Adam R, McMaster P, O'Grady JG, Castaing D, Klempnauer JL, Jamieson N et al. Evolution of liver transplantation in Europe: report of the European Live Transplant Registry. Liver Transpl. 2003; 9: 1231-1243.

10. Pappo O, Ramos H, Starzl TE, Fung JJ, Demetris AJ. Structural integrity and identification of causes of liver allograft dysfunction occurring more than 5 years after transplantation. Am J Surg Pathol. 1995; 19: 192-206.

11. Singh AK, Nachiappan AC, Verma HA, Uppot RN, Blake MA, Saini S, et al. Postoperative imaging in liver transplantation: what radiologists should know. Radiographics. 2010; 30: 339-351.

12. Sacchetto P, Veltri A, Cena V, Gisolo F, Gennari F, Salizzoni M, et al. The radiologist's role in managing paediatric liver transplantation: personal experience with 40 patients. Radiol Med. 2007; 112: 456-471.

13. Maceneaney PM, Malone DE, Skehan SJ, Curry MP, Miller JC, Gibney RG, et al. The role of hepatic arterial Doppler ultrasound after liver transplantation: an 'audit cycle' evaluation. Clin Radiol. 2000; 55: 517-524.

14. Ng S, Tan KA, Anil G. The role of interventional radiology in complications associated with liver transplantation. Clin Radiol. 2015; 70: 1323-1335

15. Hossary SH, Zytoon AA, Eid M, Hamed A, Sharaan M, Ebrahim AA. MR cholangiopancreatography of the pancreas and biliary system: a review of the current applications. Curr Probl Diagn Radiol. 2014; 43: 1-13

16. Monti L, Soglia G, Toma P. Imaging in pediatric liver transplantation. Radiol Med. 2016; 121: 378-390.

17. Wibmer A, Aliya Q, Steininger R, Trauner M, Maresch J, Mühlbacher F, et al. Liver transplantation: impaired biliary excretion of gadoxate is associated with an inferior 1-year retransplantation-free survival. Invest Radiol. 2012; 47 353-358.

18. Geisel D, Ludemann L, Hamm B, Denecke T. Imaging-Based Liver Function Tests--Past, Present and Future. Rofo. 2015; 187: 863-871.

19. Bastati N, Wibmer A, Tamandl D, Einspieler H, Hodge JC, Poetter-Lang S, et al. Assessment of Orthotopic Liver Transplant Graft Survival on Gadoxetic Acid-Enhanced Magnetic Resonance Imaging Using Qualitative and Quantitative Parameters. Invest Radiol. 2016.
20. Van Beers BE, Pastor CM, Hussain HK. Primovist, Eovist: what to expect? J Hepatol. 2012; 57: 421-429

21. Ba-Ssalamah A, Antunes C, Feier D, Bastati N, Hodge JC, Stift J, et al. Morphologic and Molecular Features of Hepatocellular Adenoma with Gadoxetic Acid-enhanced MR Imaging. Radiology. 2015; 277: 104-113.

22. Schuhmann-Giampieri G, Mahler M, Roll G, Maibauer R, Schmitz S. Pharmacokinetics of the liver-specific contrast agent Gd-EOB-DTPA in relation to contrast-enhanced liver imaging in humans. J Clin Pharmacol. 1997; 37: 587-596.

23. Lee NK, Kim S, Lee JW, Lee SH, Kang DH, Kim GH, et al. Biliary MR imaging with Gd-EOB-DTPA and its clinical applications. Radiographics. 2009; 29: 1707-1724.

24. Schwope RB, May LA, Reiter MJ, Lisanti CJ, Margolis DJ. Gadoxetic acid: pearls and pitfalls. Abdom Imaging. 2015; 40: 2012-2029.

25. Kolbe AB, Podberesky DJ, Zhang B, Towbin AJ. The impact of hepatocyte phase imaging from infancy to young adulthood in patients with a known or suspected liver lesion. Pediatr Radiol. 2015; 45: 354-365.

26. Yoon HJ, Jeon TY, Yoo SY, Kim JH, Eo H, Lee SK, et al. Hepatic tumours in children with biliary atresia: single-centre experience in 13 cases and review of the literature. Clin Radiol. 2014; 69: 113-119.

27. Olsen OE. Advances in pediatric oncology MRI. Acta Radiol. 2013; 54: 10301036.

28. Vasanawala SS, Lustig M. Advances in pediatric body MRI. Pediatr Radiol. 2011; 41: 549-554.

29. Meyers AB, Towbin AJ, Geller JI, Podberesky DJ. Hepatoblastoma imaging with gadoxetate disodium-enhanced MRI--typical, atypical, pre- and posttreatment evaluation. Pediatr Radiol. 2012; 42: 859-866.

30. Lauenstein T, Ramirez-Garrido F, Kim YH, Rha SE, Ricke J, Phongkitkarun $\mathrm{S}$, et al. Nephrogenic systemic fibrosis risk after liver magnetic resonance imaging with gadoxetate disodium in patients with moderate to severe renal impairment: results of a prospective, open-label, multicenter study. Invest Radiol. 2015; 50: 416-422.

31. Gschwend S, Ebert W, Schultze-Mosgau M, Breuer J. Pharmacokinetics and imaging properties of Gd-EOB-DTPA in patients with hepatic and renal impairment. Invest Radiol. 2011; 46: 556-566.
Austin J Radiol - Volume 4 Issue 1 - 2017

ISSN : 2473-0637 | www.austinpublishinggroup.com

Ba-Ssalamah et al. (C) All rights are reserved
Citation: Gungoren FZ, Hodge JC, Hayat A, Bastati N, Gehan M and Ba-Ssalamah A. Assessment of Liver Transplant Grafts on Gadoxetic Acid Enhanced-MRI in Pediatric Patients. Austin J Radiol. 2017; 4(1): 1066. 